\title{
El Apego en la Escuela Infantil: Algunas Claves de Detección e Intervención
}

\section{Attachment and Preeschool: Keys to Detection and Intervention}

\author{
Purificación Sierra García \\ Universidad Nacional de Educación a Distancia, España
}

\author{
Javier Moya Arroyo \\ Colegio Solynieve - C.E.S, España
}

\begin{abstract}
Resumen. Uno de los aspectos clave del desarrollo es la vinculación afectiva de apego. Ya que el desarrollo es un fenómeno integrado la naturaleza del apego tiene repercusiones en todas las demás áreas del desarrollo. El vínculo de apego ha de interpretarse como un factor de primer orden en el desarrollo y su alteración, por tanto, no puede ser vista únicamente como subsidiaria de otras alteraciones. Las alteraciones infantiles de la vinculación afectiva tienen un entorno privilegiado de expresión en la Escuela Infantil, con la maestra, las tareas y los iguales ya que, es en este contexto donde los niños (fundamentalmente desde los tres años) pasan la mayor parte del tiempo. Es esencial conocer y reivindicar el papel del maestro como figura de apego y el compromiso de toda la comunidad educativa en su implicación en esta tarea.

Palabras clave: apego, escuela infantil, maestro, atención temprana.
\end{abstract}

\begin{abstract}
One of the most important aspects in the development of a child is the emotional bonding of attachment. Because this development is considered to be an integrated phenomenon, the type of attachment developed affects all other areas of development. The bond of attachment must be considered to be the main factor in the child's development and change. Therefore, it cannot be seen as subsidiary to other alterations. The main context of disorders of emotional attachment in children is that of the nursery school (with teacher, homework and classmates). This is because at the age of 3 , this is the place where children spend most time. Therefore, it is very important to point out and emphasize the teacher's role as an attachment figure and encourage the whole of the school's staff to become involved in this task.

Keywords: attachment, preschool, teacher, early attention.
\end{abstract}

\section{Introducción}

El desarrollo es un proceso integrado y multideterminado, que ha de ser entendido desde una perspectiva sistémica y ecológica ya que por una parte, todos los ámbitos (cognitivo, afectivo, social, motor) se encuentran íntimamente ligados. Por otra, porque el desarrollo se produce en contexto, entendiendo como tal, todos los agentes y entornos físicos y sociales de crianza y aprendizaje. Por último, porque tanto ámbitos como contextos se influyen mutuamente. Por ello, cualquier alteración o riesgo en un ámbito o en un contexto, comprometen el desarrollo óptimo de los niños ${ }^{1}$.

Contar con una o varias figuras que nos hagan sentir queridos, seguros y protegidos, es una necesidad básica del ser humano (Bowlby, 1969/1982). Esta sensación de seguridad (física y psicológica) se construye sobre el establecimiento de un adecuado vínculo afectivo de apego, fruto de las repetidas interacciones entre

Purificación Sierra García pertenece al Departamento de Psicología Evolutiva y de la Educación. Facultad de Psicología de la Universidad Nacional de Educación a Distancia, UNED. Javier Moya Arroyo es el Orientador del Colegio Solynieve - C.E.S.

La correspondencia sobre este artículo debe enviarse a la primera autora al e-mail:psierra@psi.uned.es el niño y su figura de apego ${ }^{2}$ a lo largo de los tres primeros años. La calidad de dicho vínculo se ve afectada por un gran número de factores (del niño, de la madre y del contexto) teniendo repercusiones en todos los ámbitos y a lo largo de toda la vida (López, 1990, 1993, 2003; Heese y Main, 2000).

Los niños, inmersos en relaciones tempranas de apego inseguro o alterado, construyen una imagen de sí mismos como personas incompetentes, no merecedoras de cuidado y protección. Ello les lleva con frecuencia a retrasos en las adquisiciones evolutivas y a comportamientos desajustados ante la propia maestra, los iguales y las tareas que, raramente, son interpretadas como síntomas o indicios de alteraciones en el ámbito afectivo de apego. Ello a pesar de que la vinculación de apego es interpretada como un factor de protección o riesgo desde la Atención Temprana.

Tradicionalmente, el entorno fundamental de de-

\footnotetext{
${ }^{1}$ A lo largo del texto utilizamos el genérico "niño/s" para referirnos tanto a niño/s como niña/s.

${ }^{2}$ Siguiendo a Bowlby (1969) utilizamos el concepto de Figura de Apego/Cuidador Principal y madre de manera indistinta. Entendiendo que hacemos referencia, en cualquiera de los casos, a la figura adulta con la que el niño mantiene una interacción diádica y afectiva de naturaleza específica, frente a otras interacciones o relaciones.
} 
sarrollo temprano ha sido la familia y, en su seno, las interacciones entre la madre y el bebé. Sin embargo, durante las últimas décadas, la Escuela Infantil tiene, cada vez más, un papel predominante como entorno de desarrollo en los primeros años de la vida, etapa decisiva en la génesis de las relaciones afectivas de apego.

Por ello, es necesario reivindicar el papel de la Escuela Infantil como contexto de desarrollo afectivo temprano y de la maestra como figura de apego, capaz de generar oportunidades de desarrollo óptimo.

El establecimiento de un apego seguro con la maestra no sólo favorece a los niños con una historia afectiva sana, sino que lo hace, en mayor medida, a aquellos niños que por características propias, de su madre o del contexto, tienen historias afectivas de apego inseguro (p.e. Pianta, 1990 o Silver, Measelle, Armstrong y Essex, 2005).

La detección e intervención preventiva y precoz, tanto en los procesos de alteraciones del desarrollo como en los factores de riesgo que pudieran comprometer un progreso personal y social óptimo (marco conceptual de la Atención Temprana), es competencia y responsabilidad de muy diferentes agentes y ámbitos (Salud, Educación, Políticas Sociales, Agentes Económicos, etc.), todos ellos implicados en garantizar el desarrollo integral de los niños.

La consecución del objetivo de la Escuela como un contexto de oportunidades de desarrollo, requiere la tarea conjunta de profesionales sensibilizados de diferentes ámbitos. Es necesario que los distintos profesionales de la Atención Temprana, interpreten el ámbito afectivo como un aspecto clave del desarrollo. Sólo así, seremos capaces de encontrar y dar sentido cabal a actitudes y comportamientos infantiles, que habitualmente se etiquetan bajo el nombre de otras alteraciones o trastornos y en los que, por tanto, no se interviene de una manera adecuada, con el consiguiente riesgo para el desarrollo de una población absolutamente dependiente de los adultos que le rodean.

El objetivo de este artículo es contribuir a poner en valor el papel de las relaciones afectivas de apego como un elemento central del desarrollo temprano y, por tanto, no sólo como un factor subsidiario de alteraciones o riesgos para el desarrollo. En este sentido, entendemos el apego alterado (instaurado o con riesgo de hacerlo) como objeto de detección e intervención temprana por todos los agentes implicados en la misma. En este artículo, nos centramos en el contexto escolar temprano y, más concretamente, en la figura del maestro de Educación Infantil. Sin embargo, es necesario aclarar que, para nosotros, resulta imprescindible que la detección e intervención en el aula, en éste como en otros aspectos del desarrollo, se lleve a cabo de manera multidisciplinar e integrada entre todos los responsables de la institución Educativa. De esta manera, los Psicólogos Educativos, junto con los maestros han de poder fijar, a través de las claves de detección e intervención, estrategias de observación y abordaje de las alteraciones afectivas en el aula.
A lo largo de este artículo presentamos, en primer lugar, un recorrido por algunas nociones claves del concepto de apego y su relevancia en el desarrollo. Se muestra después la trascendencia de la Escuela Infantil como contexto de desarrollo temprano a la luz de los datos de escolarización en esta etapa de la vida, para pasar a realizar una breve revisión sobre los estudios relacionados con el papel de la maestra de Educación Infantil como figura de apego. A continuación, se presentan algunas claves de detección e interpretación de comportamientos infantiles en el aula, así como algunos elementos de intervención organizados en torno a la tipología clásica de apego para finalizar con un apartado de conclusiones generales.

\section{Algunas premisas sobre el apego}

Las experiencias sociales tempranas, especialmente las que se producen en el entorno familiar, resultan un elemento esencial del desarrollo emocional. El epicentro de tales experiencias sociales es la vinculación afectiva entre la madre/padre y el bebé.

La vinculación afectiva de apego, es definida como un lazo afectivo especial entre el niño y su cuidador principal, fruto de las interacciones repetidas entre ambos. Dicho lazo tiene una base biológica e innata (el mantenimiento de la supervivencia del bebé) (Bowlby, 1969) y partiendo de la misma, se modula dependiendo de factores individuales y contextuales. La vinculación afectiva de apego se organiza a lo largo de los tres primeros años de vida, se consolida a lo largo de la infancia y sus efectos se manifiestan en todos los ámbitos y a lo largo del ciclo vital (López, 1990, 1993, 1999; Heese y Main, 2000; Scott y Babcok, 2010; Sierra, Carrasco, Moya y del Valle, 2011).

Apego no es igual a apego seguro, de hecho, la cualidad de la vinculación afectiva depende del grado de ajuste, coherencia y consistencia entre las expresiones de las demandas de seguridad física y psicológica del niño y las respuestas de la figura de apego ante estas demandas (Bowlby, 1980; Isabella, 1993, 1998). Desde los dos años, el incremento de las experiencias socio-emocionales y el desarrollo de las capacidades cognitivas, hacen que las relaciones afectivas con la figura de apego pasen de un plano conductual a un plano representacional (Sierra 2006). Esta es la esencia de lo que Bowlby denomina Modelo Interno de Trabajo y que resulta la representación mental infantil de la relación afectiva. Se compone, al menos, de los siguientes elementos: a) una imagen de la relación, de sí mismo y de los otros (como seres emocionales y sociales); y b) guías para interpretar las relaciones y la actuación social en otros contextos a lo largo de la vida. Por tanto, la experiencia infantil acerca de la accesibilidad de su figura de apego, genera expectativas, atribuciones, ideas, acerca de la conducta de los otros y de sí mismo en las relaciones interpersonales (Cantón y Cortés, 2000). Dependiendo del grado de ajus- 
te entre las demandas del niño y la respuesta de su cuidador principal, se identifican varios tipos de Apego: Apego seguro, Apego inseguro evitativo, Apego inseguro ambivalente y Apego inseguro desorganizado (Ainsworth y Witting, 1969; Ainsworth y Bell, 1970; Crittenden, 1992; Main y Solomon, 1986, 1990; Main y Cassidy, 1988; Main, Kaplan y Cassidy, 1985).

La naturaleza de las relaciones de apego (seguro/inseguro) inciden en aspectos fundamentales del desarrollo psicosocial como los problemas de conducta internos y externos (Cicchetti y Toth, 2006 ó Smeekens, Riksen-Walraven y Van-Bakel, 2009). Si las primeras relaciones del niño con su cuidador son modelos negativos de interacción (hostilidad, rechazo), el niño desarrolla expectativas negativas sobre las relaciones interpersonales y, consecuentemente, desarrolla un mayor número de conductas inadecuadas (Cassidy et al., 2005; Lyons-Ruth, 1996; Lyons-Ruth y Jacobvitz, 1999; Oppenheim, Goldsmith, y KorenKarie, 2004; Steele, et al., 2007). El niño aprende a interpretar la hostilidad como un modelo válido de relación interpersonal, desarrolla expectativas de rechazo sobre los otros y configura una manera de interaccionar con el mundo. En este sentido, se han realizado investigaciones con el fin de establecer la relación entre familias de riesgo social y la desadaptación infantil. Lila y Gracia (2005) mostraron que los niños rechazados tienen numerosas alteraciones tanto a nivel interiorizado (ej. pasividad, apatía, retraimiento social, sentimientos depresivos, conductas autodestructivas) como exteriorizado (ej. impulsividad, falta de control, comportamiento violento). Moore y Pepler (2006) constataron que la manifestación de hostilidad de las figuras de apego suelen tener efectos más potentes en los niños que pertenecen a familias en riesgo social. Por tanto, en esta línea, Marcus y Kramer (2001) encuentran que los niños con problemas exteriorizados manifiestan un apego inseguro con sus madres. Troy y Sroufe (1987) concluyeron que los niños con apego evitativo tienden a percibir a sus compañeros como potencialmente hostiles $\mathrm{y}$, por consiguiente, a ser agresivos con ellos. Por su parte, LyonsRuth, Alpern y Repacholi (1993) encuentran que los niños con apego desorganizado en la primera infancia tienen mayor probabilidad de desarrollar comportamientos hostiles en el contexto escolar temprano.

Como sabemos, determinadas características individuales de los niños o aquellos que viven en contextos alterados (vulnerabilidad mental de los padres, violencia familiar o con deprivación extrema), tienen mayor probabilidad de generar apegos inseguros.

\section{La escuela infantil como contexto de desarrollo afectivo}

Durante las últimas décadas, el número de niños escolarizados en las dos etapas de Educación Infantil ha ido aumentando progresivamente. Según el último informe del Ministerio de Educación (Datos y Cifras, curso escolar 2010-2011) la tasa neta de escolarización de los niños de un año se sitúa alrededor del 25\%; sube al $43 \%$ a los dos años de edad, llegando al $98 \%$ cuando hablamos de la tasa de niños escolarizados a los 3 años.

Estos datos reflejan una realidad incontestable: la Escuela Infantil se erige, cada vez más, como contexto de desarrollo temprano.

Desde la conciencia de la importancia de la atención a los niños en edades tempranas del desarrollo, las diversas Administraciones marcan objetivos mínimos en cuanto a las capacidades, conocimientos y habilidades que los niños deben adquirir y desarrollar durante su etapa de Escolarización Infantil.

Como no podía ser de otro modo, el desarrollo afectivo y socio personal de los niños de estas edades es contemplado en todas normativas y forma parte del currículum de esta etapa. En las que se señala que "los métodos de trabajo en ambos ciclos se basarán en las experiencias, en la actividad infantil y en el juego, y se aplicarán en un ambiente de seguridad, afecto y confianza_para potenciar la autoestima y la integración, favoreciendo las creación de nuevos vínculos y relaciones" (ORDEN ECI/3960/2007, de 19 de diciembre, por la que se establece el currículo y se regula la ordenación de la educación infantil, publicada en el BOE $\mathrm{n}^{\circ} 5$, de 5 de enero de 2008).

De esta manera, el desarrollo afectivo es interpretado en dos niveles. Por una parte, como un ámbito indiscutible y esencial de progreso personal y social y, por otra, como una "condición de contexto" para dicho progreso. Es necesario aclarar y poner el acento en que, fundamentalmente, en la etapa de Escuela Infantil, ambos planos se encuentran íntimamente relacionados. Es necesario que todos los agentes implicados en el desarrollo y la educación infantil tengan claro que en esta etapa el activo central presente y futuro del niño es su mundo emocional $\mathrm{y}$, en concreto, su necesidad de sentirse querido y seguro con y de los adultos que le rodean y, para lo que a nosotros nos interesa, con y de su maestra. La Escuela es vivida por los niños (y, por tanto, ha de ser interpretada por los adultos) como un contexto de afecto.

\section{EI papel afectivo de la maestra}

Aunque la noción de apego supone una relación diádica en la que el niño establece una relación afectiva especial con la persona que ejerce de cuidador principal. Fruto del desarrollo y/o de las características del contexto de crianza, los niños van ampliando su entorno social y afectivo, de manera que aparecen otras figuras de apego. Hacia los diez meses, el mundo afectivo del niño se expande y permiten que aparezca lo que Bowbly (1969) denomina "figuras de apego 
subsidiarias". Este concepto se refiere a que, además de la figura del cuidador principal, el niño puede encontrar (siempre que se den las condiciones necesarias) en otras personas una fuente de seguridad y confort.

Este concepto de apego subsidiario es retomado por otros autores como Thompson (1999) o van Ijzendoor, Sagi y Lambernonn (1992) para enmarcar la noción de Red de Apego. Dicha Red describe el mundo afectivo del niño mediante la representación de éste como un entramado emocional y afectivo en el que el centro es ocupado por la madre o el cuidador principal, pero en el que también se encuentran otras figuras emocionalmente relevantes para el niño, especialmente aquéllas con las que tiene oportunidad constante de interactuar tanto en el tiempo como en el espacio. Estas figuras, aún con características de intensidad e interacciones contextualizadas diferentes a las que se producen en el contexto diádico y familiar, cumplen también un papel de provisión de cuidado y afecto que tiene como consecuencia la sensación del niño de seguridad física y emocional.

El tiempo que pasan los niños en las aulas de la Escuela Infantil hace que la maestra sea un adulto de referencia durante la primera infancia con características propias de figura de apego, tal y como muestra el trabajo de van Ijzendoorn et al. (1992) con niños de entre tres y cinco años. Los autores utilizan la estrategia de Ainsworth y Witting (1969) en la que se observa la naturaleza afectiva del vínculo entre el niño y la madre en función de la capacidad de ésta para ser vivida como una base segura de refugio ante una situación amenazante (la presencia de un extraño, la ausencia de la madre) y su capacidad para procurar consuelo (cuando la madre regresa). Los resultados muestran que, efectivamente, los niños mostraban un comportamiento diferencial entre el maestro y el extraño y que dichos comportamientos eran de naturaleza muy similar a los exhibidos en la situación extraña respecto a la madre y el extraño.

Cuando el maestro comienza a formar parte de la red emocional y afectiva del niño, éste tiene ya, o está construyendo (dependiendo de su edad) una vinculación afectiva de apego con su madre. Esto quiere decir que los niños tienen ya una serie de expectativas acerca de las interacciones con los adultos y las demandas del entorno y se comportan de acuerdo a dichas expectativas. Los niños, que han generado apegos seguros, muestran una orientación más positiva hacia figuras de apego alternativas en el periodo preescolar (DeMulder, Denham, Schmidt y Mitchell, 2000). Los niños, que han generado un apego inseguro con sus cuidadores principales (lo que significa, como hemos dicho, tener una imagen de sí mismo como alguien no merecedor de cariño, a sentirse sólo o desprotegido física o emocionalmente, a ver a su figura de apego como alguien indiferente ante sus necesidades, inconsistente en sus respuestas o abiertamente hostil), van a vivir la escuela, la maestra, los otros niños y las tareas como un contexto amenazante.

La noción de apego incluye la posibilidad de matizar dichas expectativas, lo que resulta clave en niños que por sus características, las de sus figuras de apegos o su entorno de crianza, han generado un apego inseguro o están en situación de riesgo de padecerlo. Bowlby (1980) o Crittenden (2002) señalan, a este respecto, que nuevas experiencias afectivas sanas pueden amortiguar o incluso reconfigurar un modelo interno inseguro, bajo la condición de que el adulto muestre una sensibilidad consistente ante las demandas de afecto del niño.

En este sentido, diversas investigaciones muestran que cuando el maestro se muestra sensible, los niños con apegos seguros con sus madres tienden a generar relaciones seguras con sus maestras tal y como era esperable. Sin embargo, el resultado más relevante es que, aunque los niños con apegos inseguros trasladan sus inquietudes e inseguridades a su relación con la maestra y con el entorno de la Escuela, la mayor parte de los niños generan apegos seguros con sus maestros cuando éstos se muestran accesibles y sensibles (p.e. Silver et al. 2005; Howes y Matheson, 1992 ó Goossens y van Ijzerdoorn, 1990). La relación afectiva segura con el maestro puede, sino remodelar la relación con la figura de apego, sí construir un espacio psicológico y afectivo de compensación donde encontrarse seguro y confiado. Desde esta premisa, una relación segura con el maestro se convierte en un factor protector para los niños con apegos inseguros o con riesgo de padecerlos (Howes y Ritchie, 1999, Carrillo, Maldonado, Saldarriaga, Vega y Díaz, 2004 o Maldonado y Carrillo, 2006). Además, la construcción de un apego seguro con la maestra en la etapa de Educación Infantil, se relaciona con mayor ajuste social y afectivo a los iguales y a los maestros en todos los niveles educativos (Howes y Ritchie, 2002; Crosnoe, Johnson y Elder, 2004; Davis y Dupper, 2004; o Silver et al. 2005) y un mayor rendimiento y actitud positiva ante las tareas y demandas escolares (Kidwell et al. 2010). Es decir, se observa una tendencia a mantener el estilo de relación afectiva con maestros en etapas posteriores de la vida y las actitudes hacia el contexto escolar y todos sus elementos y agentes (p.e. Howes, Phillipsen y Peisner-feinberg, 2000).

Parece pues, esencial que los niños, en sus primeras etapas de escolarización, establezcan unas relaciones afectivas ajustadas con la maestra. No sólo porque resultarán una "reserva emocional" sino también porque, como hemos visto, dicha relación es capaz de matizar y amortiguar una situación de partida de desventaja. Una adecuada comprensión por parte del maestro del lenguaje emocional del niño, de sus comportamientos y actitudes en el aula, con él, los iguales y las tareas, así como poseer las herramientas básicas de intervención, dentro de un trabajo en equipo, son 
elementos claves de una adecuada actuación en el aula que consiga ser un auténtico dinamizador del desarrollo infantil. En el siguiente epígrafe mostramos, de manera general, algunos indicadores que ayuden al maestro a comprender el comportamiento infantil y a actuar para favorecer el progreso en ámbito del desarrollo.

\section{¿Qué nos dicen los comportamientos infantiles sobre las necesidades de afecto de los niños? Algunas claves de interpretación e intervención}

La Escuela, el aula, el maestro, los demás niños y las tareas son contextos donde el niño se desarrolla pero también donde se refleja su mundo emocional y afectivo, tal como hemos dicho.

Las situaciones cotidianas de interacción y demandas típicas de la vida del aula, nos aportan indicadores muy valiosos acerca de la historia y el bagaje afectivo del niño (organizado o en construcción), y que son elementos de conocimiento esencial para una adecuada interpretación y, en su caso, intervención tanto por la maestra como por los agentes educativos pertinentes.

Siguiendo a Barret y Trevitt (1991), Howes y Ritchie (1999) o más recientemente Geddes (2006), vamos a presentar los comportamientos infantiles en torno a: a) las interacciones con la maestra; b) las interacciones con los iguales y c) las actitudes y conductas frente a las tareas, en relación con los patrones típicos de apego infantil.

\section{Niños que se sienten seguros en el aula}

Los niños que han establecido un apego seguro con sus figuras de apego o que sus madres se muestran consistentemente sensibles a sus demandas, generan una sensación de seguridad física y psicológica. Aprenden que, cuando se sienten amenazados, pueden contar con su figura de apego y que serán "escuchados, comprendidos y atendidos". Para estos niños, otros adultos pueden comportarse de la misma manera, sensible y cálida y el mundo es un lugar que explorar, lleno de oportunidades de aprendizaje. Ello no quiere decir que quieran separarse de su figura de apego, sino que otras figuras sensibles pueden ganarse su confianza y calmarles.

Estos niños tienen un comportamiento típico de desagrado y protesta ante las primeras separaciones de la figura de apego que va desapareciendo a medida que interpreta a la maestra como una figura sensible y aumenta su conocimiento del entorno (espacio físico, objetos, tareas e iguales). Una vez aceptada la separación, procuran la cercanía física y emocional con la maestra, buscándola y pidiendo ayuda de manera explícita cuando se encuentran en situaciones conflictivas o interpretan alguna situación como amenazante.
Atienden las demandas e instrucciones de la maestra y acatan las normas y las sanciones, corrigiendo su comportamiento.

Los niños seguros, tienen curiosidad por el entorno y lo exploran de manera confiada. Son niños que investigan y ensayan con los espacios y los objetos. Suelen aceptar las tareas de buen grado, tienen un nivel de tolerancia a la frustración aceptable y reaccionan ante las demandas de la maestra de manera fácil y rápida.

Las interacciones son apropiadas a su edad, mostrando una buena disposición para comenzar o entablar relaciones afectivas cálidas con los demás niños, teniendo en cuenta el desarrollo de la capacidad para establecer relaciones. Son capaces de iniciar o mantener acciones conjuntas con otros niños, en mayor medida cuanto más estructuradas y lideradas estén por la maestra.

Sus demandas son coherentes con las circunstancias, el tono de las mismas ajustado y las reacciones ante las respuestas de los demás niños y del maestro son consecuentes y esperables. Ello hace que los maestros, por una parte, interpreten de forma más adecuada y ajustada las demandas y reacciones del niño, se sientan más satisfechos con los logros tanto cognitivos como afectivos del niño y, por tanto, se generen de manera espontánea interacciones más frecuentes, de mayor calidad y duración.

Cuando el niño se reencuentra con la figura de apego suele mostrarse confiado y tranquilo, y aunque no se acerque a ella si está realizando alguna tarea interesante, realiza frecuentes contactos visuales o físicos.

\section{Niños invisibles en el aula}

Otros niños, sin embargo tienen una historia vincular diferente. Sus madres o cuidadores principales se han mostrado consistentemente insensibles y el niño ha aprendido que sus demandas de afecto y seguridad no van a ser atendidos. Estos niños, por tanto, tienden a no mostrar necesidad. Es lo que se denomina un apego evitativo.

Cuando los niños se separan de sus padres para entrar en la Escuela pueden no mostrar ningún signo de ansiedad, ni siquiera protesta. Parecen permanentemente distraídos o indiferentes. Suelen ignorar las llamadas de atención de la maestra que para ellos resulta, en un comienzo, una extensión de la figura de apego quien, como se recuerda, no atendía a sus demandas. Por ello, no es de extrañar que estos niños no reclamen la ayuda de la maestra ante situaciones conflictivas, tanto con el entorno como con los iguales o incluso pueden mostrarse molestos, alejándose del adulto, lo que refuerza la sensación de la maestra de que no es necesaria. Este "pasar desapercibido" hace que, ante la presencia y demanda constante de los demás niños, suelen ser interpretados por los adultos como niños que 
"se han adaptado muy rápidamente". Una observación más detenida de sus expresiones, movimientos y reacciones nos muestran un panorama bien distinto, si se interpreta en las claves adecuadas.

Por lo que respecta a las tareas, tienden a abordarlas de manera individual, con recelo y suelen mostrar un comportamiento de acercamiento y alejamiento, como calibrando su capacidad para llevarla a cabo. En muchos casos, comienzan la tarea pero se frustran ante la primera adversidad o incluso ante la observación de que otros niños las están realizando correctamente. Para los niños evitativos, las tareas cumplen la misión de catalizador de las relaciones interpersonales. Por ello, redirigen el miedo o la rabia hacia los objetos y las demandas de la tarea.

Los niños que han construido (o están construyendo) una relación afectiva evitativa (al igual que todos los apegos inseguros), tienden a verse a sí mismos como alguien no merecedores de atención, lo que les lleva a generar una imagen de sí mismos muy empobrecida. Suelen pensar que, al igual que no son capaces de despertar afecto tampoco lo son de ser eficientes en ningún área. Por ello, en concreto para los niños evitativos, su relación con las demandas de la maestra, las tareas escolares resultan un excelente aliado si son interpretadas como una vía de intervención.

Como hemos dicho, estos niños tienden a interpretar las tareas como un reto insuperable, algo que les puede poner en evidencia su capacidad y reafirmar con ello su sentimiento de ineficacia. Es importante, por tanto, que el maestro comprenda que los comportamientos explosivos y hostiles ante las tareas y más cuando a la vista del niño, resulta compleja. El niño puede vivir una tarea como compleja por diversas razones, objetivas o subjetivas. Algunas de las principales son, la vivencia de que: a) necesita competencias o habilidades que el niño no posee; b) son muy complejas y necesitan poner en juego diversas actividades; c) las instrucciones no le resultan claras; d) no tiene claros los objetivos ni el proceso para llegar a ellos; e) resultan demasiado largas o fatigosas.

Ya que los niños evitativos o en riesgo de generar estas relaciones afectivas con los adultos, se alejan de ellos y no piden ayuda, la maestra puede articular sus procesos de interacción a través de las tareas. La planificación de las mismas, las instrucciones de ejecución y el refuerzo positivo pueden ser aliados beneficiosos tanto para el desarrollo del niño como un facilitador de las interacciones con la maestra y los iguales.

\section{Los niños que no saben si pueden confiar}

En ocasiones, bien por las características de la madre, del niño o del entorno, las relaciones afectivas entre el niño y su figura de apego tienen como patrón de interacción la incoherencia. Estas relaciones dan como consecuencia lo que denominamos un Apego Ambivalente o Resistente. La historia o el proceso vincular de estos niños está dominado por las reacciones impredecibles de su figura de apego ante sus demandas. Estas madres tienden a reaccionar de una manera inconsistente y esas respuestas (afecto-rechazo) dependen en gran medida de factores internos (estado de ánimo, vivencia personal de la demanda del bebé, etc.). Por ello, el niño es incapaz de predecir cuándo y de qué depende el ser atendido de una manera sensible, lo que genera dos sentimientos contrapuestos: por una parte, conductas de búsqueda de apoyo y afecto de manera casi compulsiva y por otra, el rechazo y la frustración ante las reacciones de la madre. Hay que señalar que un elemento clave de este tipo de relaciones es la presencia de dificultades de separación saludable por ambos miembros de la díada, fruto de la inestabilidad y la incoherencia emocional que la caracteriza, por lo que con frecuencia ante comportamientos de llantos o aferramientos con la figura de apego en el momento de la despedida, pueden confundirse con niños seguros de su madre y, de nuevo, el conocimiento de las relaciones diádicas entre el niño y su figura de apego y la vida del niño en el aula, nos dan claves de interpretación adecuada.

Los niños resistentes suelen mostrar un comportamiento ambivalente con la maestra. Para estos niños, la maestra, su atención y la interacción, es el eje fundamental alrededor del que gira su vida y emociones en el aula. Los niños temen que la maestra resulte tan impredecible como su figura de apego y, por tanto, se encuentran desorientados respecto a cómo obtener la protección y el apoyo que necesitan, mostrando un comportamiento dependiente y a la vez mostrando su miedo y frustración con comportamientos hostiles hacia ella, incluso cuando intenta consolarles. Ello ha de ser interpretado por la maestra como una petición de ayuda y de seguridad y no como un síntoma de rechazo.

La maestra ha de organizar sus interacciones de manera diádica, de forma que el niño pueda llegar a aprender y comprender (y, por tanto, a tener una imagen) que existe una relación consistente entre sus demandas/reacciones/comportamientos y los de la maestra. En definitiva, estos niños deben aprender qué se espera de ello, cómo conseguirlo y cuál será el resultado afectivo.

Para ello, las rutinas y actividades pueden resultar unos buenos mediadores ya que el niño aprende a interpretarlas como una guía estructurada con resultados predecibles. El refuerzo, en cada fase del desempeño de la tarea y la explicitación de las acciones y consecuencias, son elementos de anclaje sobre los que el niño construirá confianza en sus actuaciones y los resultados.

Debido a la dificultad de separación respecto a su figura de apego, para estos niños es especialmente relevante la posibilidad de contar con elementos /objetos/imágenes que les hagan sentirse cerca de sus en- 
tornos conocidos. En este sentido, es esencial prestar atención a estrategias de transición entre actividades y/o rutinas, de manera que el niño pueda comprender las separaciones de los entornos (p.e. el momento de salir al patio, el cambio de actividad). Las regularidades son otro de los elementos esenciales de organización mental y afectiva del niño resistente.

Fruto de su aprendizaje, los niños parecen estar en permanente estado de alerta, de manera que cualquier acontecimiento del entorno como ruidos inesperados, movimientos de otros niños o incluso acercamientos sorpresivos de la maestra, les suele provocar llanto y reacciones exageradas pero, como hemos dicho, las estrategias de consuelo de la maestra no siempre tienen éxito. De nuevo, estos comportamientos han de enmarcarse en una historia vincular en la que el niño ha de realizar permanentemente el esfuerzo de esperar lo impredecible.

\section{El niño asustado}

Aunque resulta minoritario, hay un grupo de niños que, víctimas de abusos o de conductas claramente negligentes debido a alteraciones psicológicas de la figura de apego o de un entorno absolutamente desestructurado, generan apegos desorganizados. Las relaciones con sus figuras de apego se encuentran dominadas por el miedo y la indefensión. Mientras los niños con madres consistente o inconsistentemente insensibles tienen una cierta forma de organización mental de la relación. Estos niños viven dentro de un patrón de relación patológico.

Aprenden a mantenerse en alerta permanente bajo la premisa de la supervivencia física y psicológica. De nuevo, estos niños necesitan del afecto y la atención y, por eso, muestran conductas de acercamiento contradictorio hacia la madre (como por ejemplo, acercándose pero mirando hacia otro lado (Ainsworth y Bell, 1969). La consecuencia es un patrón de reacciones desajustadas y claramente patológico en el que aparece una elevada ansiedad, apatía con ráfagas de ira o de tristeza, reacciones incontroladas e incoherentes con los acontecimientos, recelo ante la maestra, los otros niños y las tareas, así como comportamientos dañinos e insensibles ante el dolor o las demandas de los demás (Schore, 2000).

Dado estos comportamientos, es esencial la detección temprana de la historia vincular del niño y resulta imprescindible el trabajo conjunto, organizado y planificado, de todos los responsables del entorno educativo. En estos casos, más que nunca, los niños necesitan encontrar en la maestra un refugio emocional y afectivo y entender el entorno escolar como un lugar seguro y fiable.

Acabamos de presentar algunas claves para interpretar los comportamientos de los niños e intervenir atendiendo a los diferentes patrones de apego. Además de ellas, con carácter general, conocer la historia personal y social del niño, nos dará información esencial de su situación individual y social de partida. La observación de las interacciones entre el niño y sus figura de apego en situaciones críticas como despedidas, reencuentros o momentos de interacción entre la maestra y el adulto (como tutorías o momentos de charla), nos pueden aportar información valiosa como indicios que, junto con otras señales, nos permitan dibujar un panorama general de las relaciones afectivas entre ambos. En este sentido, es necesario señalar que, como consecuencia de la inexistencia o la precariedad de su seguridad, los niños con apegos inseguros tienden a estar muy atentos a las disonancias entre los mensajes emocionales en privado y en público respecto a ellos. Es importante, por tanto, que exista coherencia entre las emociones y sensaciones que recibe de la maestra en privado, fruto de las interacciones cotidianas y las que transmite a otros niños y fundamentalmente a otros adultos, entre los que destacan los padres. Cuanto mayor sea el niño, más fácilmente captará los comentarios y descripciones que sobre él se hagan, comparándolas con el comportamiento del que es objeto. La discrepancia entre ambos, repercutirá en la confianza depositada en el maestro. Para finalizar, el entorno físico debe poder brindarle seguridad física y emocional, con espacios sencillos y abordables, actividades predecibles y rutinas coherentes y consistentes. Estas rutinas, de especial importancia para todos los niños, son especialmente relevantes para ellos porque necesitan coordenadas claras y precisas que les ayuden a autorregular su comportamiento.

\section{Conclusiones}

El desarrollo óptimo de los niños con alteraciones o riesgos de padecerlas es el marco de la Atención Temprana. Cumplir con este derecho de los niños y sus familias necesita de una visión multidisciplinar y sistémica. Multidisciplinar por los ámbitos y los agentes co-responsables (salud, educación, políticas sociales) y sistémica por la concepción del desarrollo como un entramado de ámbitos y contextos mutuamente influidos.

La Escuela Infantil, como hemos visto, es un contexto mayoritario de desarrollo temprano entre la población de niños de tres años de edad. En edades tempranas, una de las claves de desarrollo son las relaciones afectivas de apego. Dichas relaciones son interpretadas desde la Atención Temprana como factor de riesgo/protección, tanto porque los niños con alteraciones del desarrollo tienen más posibilidades de generar apegos inseguros como que las alteraciones de la vinculación afectiva son, en sí mismas, un factor de riesgo para el desarrollo.

Los niños, que han generado apegos inseguros con sus padres, pueden encontrar en la maestra la confir- 
mación de su imagen como personas no merecedoras de afecto y todo lo que ello supone de lastre para un adecuado desarrollo, o bien pueden encontrar en la escuela y la maestra un contexto y una figura de apoyo, confort y seguridad física y emocional. Parece pues, que fundamentalmente para los niños que parten con desventaja personal o social, resulta esencial el que los maestros tengan, entre sus prioridades, la construcción de un buen vínculo afectivo que se fundamenta en actitudes y comportamientos de sensibilidad y calidez en las interacciones. La escuela y la maestra, en estos casos, se convierte en un factor de protección crucial y en un aliado de desarrollo.

El conocimiento por parte del maestro de cómo se produce el desarrollo emocional y afectivo y cómo éste se encuentra afectado por circunstancias o factores alterados o con riesgo de alterarse, resulta un elemento formativo esencial que le facilitaría en gran medida la tarea de detección y comprensión del progreso infantil. Los niños, con alteraciones o contextos alterados de crianza, tienen dificultades tanto para determinar sus emociones y relacionarlas con los acontecimientos como también para expresarlas $\mathrm{y}$, por supuesto, para hacerlo de manera efectiva. Por ello, es esencial no sólo que la maestra pueda comprender en contexto el comportamiento social y emocional del niño, sino que procure un entorno de reconocimiento y etiquetado de las emociones y los acontecimientos que las provocan. Aunque la comprensión emocional es una tarea evolutiva (Harris, 1989), cuando algunos factores no favorecen la interpretación adecuada de las emociones y la regulación emocional, los niños tienen mayores dificultades para ser competentes emocionalmente (Saarni, Mumme y Campos, 1998). Al igual que la sensibilidad materna es el mejor predictor del tipo de apego (por encima de las dificultades o alteraciones del bebé o del entorno) (p.e. Isabella, 1993), la sensibilidad, receptividad e implicación personal de la maestra tienen un papel destacado en el establecimiento de relaciones de apego seguras (Howes y Ritchie, 2002) aún cuando las características o circunstancias de partida del niño resulten adversas (Barret y Trevitt, 1991).

La maestra tiene la capacidad de generar un entorno de comprensión y seguridad, donde el niño se sienta capaz y querido, donde sus avances se vivan como auténticos progresos. Tiene la capacidad, en esencia, de convertirse en una auténtica base segura a la que acudir para tomar oxígeno en la difícil travesía del aprendizaje y el desarrollo. Como señalan Pianta y LaParo (2003) el establecimiento de una relación positiva entre el niño y la maestra debe ser tomado como un aspecto clave a la hora de evaluar la calidad de un programa educativo.

Para ello, es esencial la implicación de todos los agentes educativos y su sensibilización de manera que el ámbito afectivo no quede relegado a la enseñanza de estrategias de interacción social sino que sea entendido como un verdadero motor de desarrollo.

\section{Extended Summary}

Having one or two figures that make us feel loved, safe and protected is a basic need for human beings (Bowlby, 1969/1982). The theory of emotional attachment is defined as the inner tendency to establish relationships which are emotionally meaningful and special, with one or more figures. This relationship is constructed during the first months of life and is organized at about at the age of three. It can be seen in different behaviors and attitudes which show us the nature of this relationship.

As a result of repeated interactions between mother and child, the offspring learns the relation between its demands for care and protection and the responses of the attachment figure. Thus, the consistent, sensitive and coherent response of the attachment figure to the demands of the child has positive consequences in the development of a secure attachment. On the other hand, if the mother's answers are indifferent, hostile or inconsistent, the child learns that this attachment figure is unable to give any kind of protection. This type of relationship makes the child feel as if they do not deserve any love or protection, and instead they think that the only thing that they deserve is punishment. In this learning process, adults in general are widely perceived to have the same characteristics as the attach- ment figures. These perceptions represent a disruption in the child's development, and therefore an issue for early intervention.

That nursery school is a highly important environment for early development is also true for children with affective development disorders. In these cases, it can act as a protective environment and the role of nursery school teachers as an attachment figure is crucial.

A bond of healthy attachment with the teacher should be one of the objectives for this educational stage, since one of the basic needs of the child at these ages is his/her need to feel protected and safe, both physically and psychologically.

At pre-primary school, the child is already building, or organizing, a bond of attachment with the first caregiver. This makes the child consider the environment and the teacher as an extension of this bonding situation. In this way, those children who have insecure attachment, or who have personal or social situations which put them at risk of suffering this problem, will see school, other children and school demands as hostile. This situation leads them to have disorder behaviors.

A secure emotional relationship with the teacher can 
both re-create the relationship with an attachment figure and build a psychological space and emotional compensation where the child feels safe and trusting. From this point of view, a safe relationship with the teacher becomes a protective factor for children with insecure attachment or who are at risk of suffering it (Howes, 1999; Carrillo et al., 2004; Maldonado \& Carrillo, 2006).

The establishment of secure attachment with the teacher not only favors children with a healthy emotional history, but also, and to a greater extent, those children whose attachment is insecure because of their mother or their environment (e.g. Pianta, 1990; Silver et al., 2005).

Furthermore, it should be pointed out that most children have safe attachments with their teachers, where they are accessible and sensitive (e.g. Silver et al., 2005; Howes \& Matheson, 1992; Goossens \& van Ijzerdoorn 1990).

It is essential to provide teachers with the tools and procedures which let them interpret children's behaviors in the classroom and give them strategies to act effectively. This task should not be interpreted separately, but within a set of multidisciplinary interventions of all educational factors which are involved in the task of achieving the positive development of the child.

\section{Referencias}

Ainsworth, M. D. S. y Bell, S. M. (1969). Some contemporary patterns in the feeding situation. En A. Ambrose (Ed.), Stimulation in early infancy (pp. 133-170). London: Academic Press.

Ainsworth, M. y Witting, B. A. (1969). Attachment and exploratory behavior of one years olds in a strange situation. En B. M. Foss (Ed.), Determinants of Infant behavior, (pp. 111-136). London: Methuen.

Ainsworth, M. y Bell, S. M. (1970). Attachment, exploration, and separation: Illustrated by the behavior of oneyear-olds in a strange situation. Child Development, 41, 49-67.

Barret y Trevitt (1991). Attachment Behaviour and the schoolchild: An introduction to educational Therapy. London: Routledge.

Bowlby, J. (1969/1982). Attachment and loss: Vol. 1. Attachment. New York: Basic Books.

Bolwby, J. (1980). Attachment and Loss. Vol. 3 Loss, sadness and depression. Londres: Hogarth Press. (Trad. cast. La pérdida. Buenos Aires: Paidós, 1985).

Cassidy , J., Woodhouse, S. S, Cooper, G., Hoffman, K., Powell, B. y Rodenberg, M. (2005). Examination of precursors of infant attachment security: Implications for early intervention research. En J. L. Berlin, Y. Ziv, L. Amaya-Jackson y M. T. Greenberg (Eds.) Enhancing early attachment: theory, research, interventions and policy. (pp. 34-60). New York: Guilford Press.
Cantón, J. y Cortés, Mª R. (2000). Teoría y Evaluación del Apego. En J. Cantón y $\mathrm{M}^{\mathrm{a}}$ Rosario, El apego del niño a sus cuidadores. Madrid: Alianza.

Carrillo, S. Maldonado, C. Saldarriaga, L., Vega, L y Díaz, S. (2004). Patrones de apego en familias de tres generaciones: Abuela, madre adolescente, hijo. Revista Latinoameracana de Psicología, 36, 409-430.

Cicchetti, D. y Toth, S. L. (2006). Developmental psychopathology and preventive intervention. In A. Renninger and I. Sigel (Eds.), Handbook of Child Psychology (6th Ed.) (pp. 497-547). New York: John Wiley \& Sons, Inc.

Crittenden, P. (1992). Quality of attachment in the preschool years. Developmental and Psychopathology, 4, 209-241.

Crittenden, P. (2002). Nuevas implicaciones clínica de la teoría del apego. Valencia: Promolibro.

Crosnoe, R., Johnson, M. K. y Elder, G. H. (2004). Intergenerational bonding in school: The behavioural and contextual correlates of student-teacher relationships. Sociology of Education, 77, 60-81.

Davis, K. y Dupper, D. (2004). Student-teacher relationships: An overlooked factor in school dropout. Journal of Human Behavior in the Social Environment, 9, 179-193.

DeMulder, E; Denham, S., Schmidt, M. y Mitchell, J. (2000). Q-sort assessment security during the preschool years: Links from home to school. Developmental Psychology, 36(2), 274-282.

Geddes, H. (2006). El apego en el aula. Relación entre las primeras experiencias infantiles, el bienestar emocional y el rendimiento escolar. Graó. Barcelona (2010).

Goossens, F. A. y Van Ijzendoorn, M. H. (1990) Quality of infant's attachments to professional caregivers: Relation to infant-parent attachment and day care characteristics. Child Development, 61, 832-837.

Harris, P. (1989). Children and emotion. The development of psychological understanding. Oxford: Basil Blackwell. Trad. cast. Los niños y las emociones. Madrid: Alianza, 1992.

Heese, E. y Main, M. (2000). Disorganized infant, child and adult atachment: Collapse in behavioral and attentional strategies. Journal of the American Psychoanalytic Association, 48 (4), 1097-1127.

Howes, C. (1999). Attachment relationships in the context of multiple caregivers. En J. Cassidy y P. R. Shaver (Eds.), Handbook of attachment. Theory, research and clinical applications (pp.671-687). New York, The Guildford Press.

Howes, C. y Matheson, C. C. (1992). Contextual constraints on the concordance of mother-child an teacher-child relationships. New Directions for Child Development, 57, 2540.

Howes, C., Phillipsen, L. C. y Peisner-feinberg, E. (2000). The consistency of perceived teacher-child relationships between preschool and Kindergarten. Journal of School Psychology, 38, 113-132.

Howes, C. y Ritchie, S. (1999). Attachment organizations in children with difficult life circumstances. Development and Psychopathology, 11, 251-268. 
Howes, C. y Ritchie, S. (2002). A Matter of Trust: Connecting Teachers and Learners in the Early Childhood Classroom. NY: teacher College Press.

Isabella, R.A. (1993). Origins of Attachment: maternal interactive behavior across the first year. Child development, 64, 605-621.

Isabella, R.A. (1998). Origins of attachment: the role of context, duration, frequency of observation , and infant age in measuring maternal behavior. Journal of Social and Personality Relationships, 15, 538-554.

Kidwell, S., Young, M., Hinkle, L., Ratliff, A, Marcus, M. y Martin, C. (2010). Clinical Child Psychology and Psychiatry, 15, 391-406.

Lila, M. y Gracia, E. (2005). Determinantes de la aceptación-rechazo parental. Psicothema, 17, 107-111.

López, F. (1990). El apego. En J. A. Madruga y P. Lacasa (Eds.) Psicología Evolutiva I. Madrid: Universidad Nacional de Educación a Distancia.

López, F. (1993). El apego a lo largo del ciclo vital. En M. J. Ortiz, S. Yárnoz (Eds.) Teoría del apego y relaciones afectivas. Bilbao: Universidad del País Vasco.

López, F. (1999). La evolución del apego desde la adolescencia hasta la muerte. En F. López, I. Etxebarría, M. J. Fuentes y M. J. Ortiz (Eds.) Desarrollo afectivo y social. (pp. 67-93). Madrid: Psicología Pirámide.

López, F. (2003). I Intervención temprana en niños de riesgo social. En A. Gómez, P. Viguer, M. J. Cantero (Coords.) Intervención temprana. Desarrollo óptimo de 0 a 6 años. (pp. 315-335). Madrid: Pirámide.

Lyons-Ruth, K. (1996). Attachment relationships among children with aggressive behavior problems: The role of disorganized early attachment patterns. Journal of Consulting and Clinical Psychology, 64, 32-40.

Lyons-Ruth, K., Alpern, L. y Repacholi, B. (1993). Disorganized infant attachment classification and maternal psychosocial problems as predictors of hostile-aggressive behavior in the preschool classroom. Child Development, 64, 572-585.

Lyons-Ruth, K. y Jacobovitz, D. (1999). Attachment disorganization: Unresolved loss, relational violence and lapses in behavioral and attentional strategies. En J. Cassidy \& P. Shaver (Eds.), Handbook of attachment theory and research. (pp. 520-554). New York: Guilford Press.

Main, M. y Cassidy, J. (1988). Categories of response to reunion with the parent at age 6: Predictable from infant attachment classifications and stable over 1-month period. Developmental Psychology, 24, 415-426.

Main, M., Kaplan, N. y Cassidy, J. (1985). Security in infancy, childhood, and adulthood: A move to the level of representation. In I. Bretherton, E. Waters, (Eds.), Growing points in attachment theory and research. Monographs of the Society for Research in Child Development, 50 (1-2, Serial No. 209), 66-104.

Main, M. y Solomon, J. (1986). Discovery of an insecuredisorganized/desoriented patters. En T. B. Brazelton y M. W. Yogman (Eds.), Affective development in infancy. Norwood, NJ: ABLEX, 95-124.

Main, M. y Solomon, J. (1990). Procedures for identifying infants as disorganized/disoriented during the Ainsworth Strange Situation. En M. T. Greenberg, D. Cicchetti y E. M. Cummings (Eds.), Attachment in the preschool years (pp. 121-160). Chicago: Chicago University Prees.

Maldonado, C. y Carrillo, S. (2006). Educar con afecto: Características determinantes de la calidad de la relación niño-maestro. Revista Infancia Adolescencia y Familia, 1(1), 39-68.

Marcus, R. F. y Kramer, C. (2001). Reactive and proactive aggression: Attachment and social competence predictors. The Journal of Genetic Psychology, 162(3), 260-275.

Ministerio de Educación. Datos y Cifras, curso escolar 20102011.

Moore, T. E. y Pepler, D. J. (2006). Wounding words: Maternal verbal aggression and children's adjustment. Journal of Family Violence, 21, 89-93.

Oppenheim, D., Goldsmith, D. y Koren-Karie, N. (2004). Maternal Insightfulness and preschoolers' emotion and behavior problems: Reciprocal influences in a daytreatment program. Infant Mental Health Journal, 25, 352367.

ORDEN ECI/3960/2007, de 19 de diciembre, por la que se establece el currículo y se regula la ordenación de la educación infantil. BOE $\mathrm{n}^{\circ} 5$, de 5 de enero de 2008, 10161036.

Pianta, R.C. (1990). Widening the debate on educational reform. Prevention as a viable alternative. Exceptional Children, 59, 306-313.

Pianta y LaParo (2003). Improving early school success. Baltimore: Paul Brookes Publishing. Co.

Saarni, C., Mumme, D. L. y Campos, J. J. (1998). Emotional development: Action, comunication and understanding. En W. Damon y N. Eisenberg (Eds.) Handbook of child psychology (5th ed. Vol. 3, pp. 237-310). Social, emotional and personality development. N. Y: Wiley \& Sons.

Scott, S. y Babcock, J.C. (2010). Attachment as a moderator between intimate partner violence and PTSD symptoms. Journal Family Violence, 25, 1-9.

Sierra, P. (2006). La génesis y desarrollo de las primeras experiencias socioafectivas. En P. Sierra, P. y A. Brioso, Psicología del desarrollo. Introducción al cambio evolutivo. (pp. 97-129 ). Madrid: Sanz y Torres. Madrid.

Sierra, P., Carrasco, M. A., Moya, J. y del Valle, C. (2011). Entrevista de apego para Niños (EAN): Estudio exploratorio de un nuevo instrumento de evaluación del apego en población infantil de 3 a 7 años. Acción Psicológica, 8 , (2), 39-53.

Silver, R. B., Measelle, J. R., Armstrong, J. M. y Essex, M. J. (2005). Trajectories of classroom externalising behavior: Contributions of child characteristics, family characteristics, and the teacher-child relationship during the school transition. Journal of School Psychology, 43, 39-60.

Schore, A. (2000) The effects of early relational trauma on right brain development, affect regulation and infant mental health. Mental Health Journal, 22, 201-269.

Smeekens, S., Riksen-Walraven, J. M. y Van Bakel, H. J. (2009). The predictive value of different infant attachment measures for socioemotional development at age 5 
years. Infant Mental Health Journal, 30, 366-383. doi:10.1002/imhj.20219

Steele M., Hodge, J., Kaniuk, J., Steele, H., D`agostino, D., Blom, I., ... Henderson, K. (2007). Intervening with maltreated children and their adoptive families. Identifying attachment-facilitative behaviors. En D. Oppenheimer y D. Goldsmith, (Eds.) Theory in clinical work with children. Bridging the gap between research and practice. (pp. 58-89). New York: Guilford Press.Guilford Press.

Troy, M. y Sroufe, L. A. (1987). Victimization among preschoolers: The role of attachment relationship history. Journal of the American Academy of Child and Adolescent Psychiatry, 26(2), 166-172.

Thompson, R. A. (1999). Early attachment and later development. En J. Cassidy y P. R. Van Ijzendoord, M. H., Sagi, A. M. y Lanbernon, W. E. (1992). The Multiple Caretaker Paradox: Data from Holland and Israel. En R. C. Pianta (Ed). Beyond the Parents: The Role of Other Adults in Chldren's Lives. (pp. 5-24). San Francisco: Jossey-Bass Publisher.

Manuscrito recibido: 30/03/2012

Revisión recibida: 19/10/2012

Manuscrito aceptado: 29/10/2012 